\title{
Equation of state of charged colloidal suspensions and its dependence on the thermodynamic route
}

Cite as: J. Chem. Phys. 136, 194103 (2012); https://doi.org/10.1063/1.4718367

Submitted: 21 March 2012 . Accepted: 30 April 2012 . Published Online: 16 May 2012

Thiago E. Colla, Alexandre P. dos Santos, and Yan Levin

\section{ARTICLES YOU MAY BE INTERESTED IN}

Interaction between random heterogeneously charged surfaces in an electrolyte solution

The Journal of Chemical Physics 142, 194707 (2015); https://doi.org/10.1063/1.4921410

Simulations of ionic liquids confined by metal electrodes using periodic Green functions

The Journal of Chemical Physics 147, 074109 (2017); https://doi.org/10.1063/1.4989388

Equilibrium properties of charged microgels: A Poisson-Boltzmann-Flory approach

The Journal of Chemical Physics 141, 234902 (2014); https://doi.org/10.1063/1.4903746

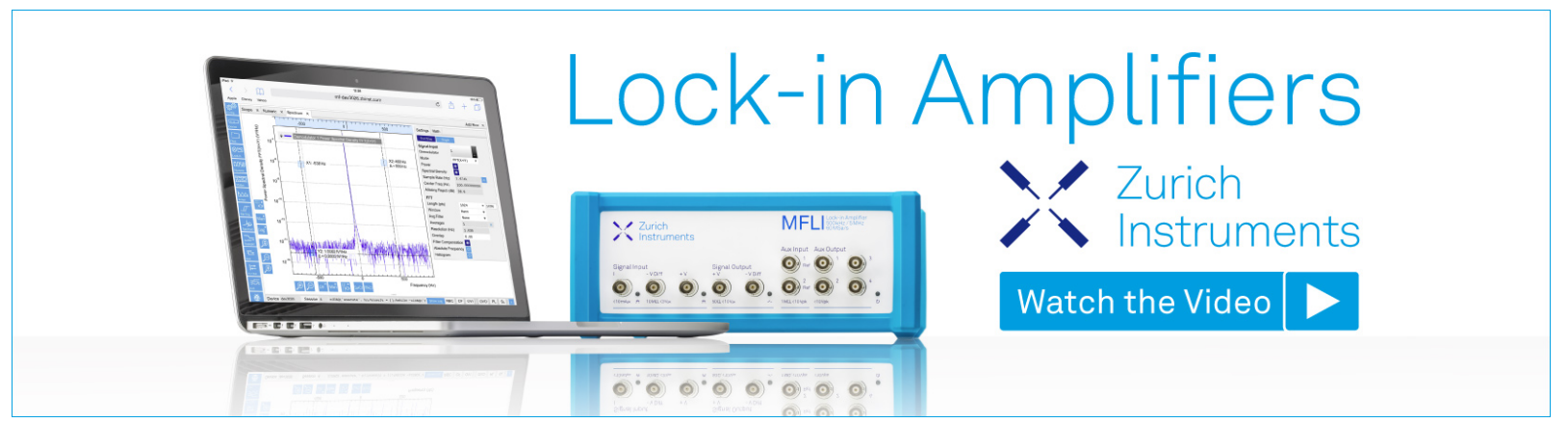

J. Chem. Phys. 136, 194103 (2012); https://doi.org/10.1063/1.4718367 


\title{
Equation of state of charged colloidal suspensions and its dependence on the thermodynamic route
}

\author{
Thiago E. Colla, Alexandre P. dos Santos, and Yan Levin \\ Instituto de Física, Universidade Federal do Rio Grande do Sul, CP 15051, 91501-970 Porto Alegre, RS, Brazil
}

(Received 21 March 2012; accepted 30 April 2012; published online 16 May 2012)

\begin{abstract}
The thermodynamic properties of highly charged colloidal suspensions in contact with a salt reservoir are investigated in the framework of the renormalized Jellium model (RJM). It is found that the equation of state is very sensitive to the particular thermodynamic route used to obtain it. Specifically, the osmotic pressure calculated within the RJM using the contact value theorem can be very different from the pressure calculated using the Kirkwood-Buff fluctuation relations. On the other hand, Monte Carlo simulations show that both the effective pair potentials and the correlation functions are accurately predicted by the RJM. It is suggested that the lack of self-consistency in the thermodynamics of the RJM is a result of neglected electrostatic correlations between the counterions and coions. (C) 2012 American Institute of Physics. [http://dx.doi.org/10.1063/1.4718367]
\end{abstract}

\section{INTRODUCTION}

In spite of its fundamental importance-both practical and theoretical - the thermodynamic properties of charged colloidal suspensions are far from understood. ${ }^{1-4}$ Even such basic question as the existence of a liquid-gas phase transition in these systems still remains a topic of debate..$^{5-8}$ The difficulty in describing the thermodynamics of charged colloidal suspensions is a consequence of both size and charge asymmetry between the different components of the system and the long-range nature of the Coulomb interaction. . $^{1,9,10}$ To simplify the theoretical description one often uses the, so-called, primitive model (PM). In this model all charged components-colloidal particles, coions, and counterionsare treated explicitly, while the solvent-usually an aqueous medium-is considered as a dielectric continuum. The interactions between the colloidal particles, the counterions, and the coions have both Coulomb and hard-core components. Image effects resulting from the dielectric discontinuities across the particle surface are usually neglected at the lowest order of approximation. ${ }^{11-15}$

Colloidal suspensions often contain salt. For theoretical description it is, therefore, convenient to work in a semigrand-canonical ensemble in which the number of colloidal particles is fixed, while the concentration of salt is controlled by an externally imposed chemical potential. Physically this can be realized by separating the suspension from a salt reservoir by a semi-permeable membrane transparent only to microions. ${ }^{16,17}$

The large asymmetry between the colloidal particles and the microions forces us to employ different approximations to account for the correlations among the various components of suspension. The correlations among the microions can be described by a linear Debye-Hückel (DH) like theory. For dilute colloidal suspensions these correlations are usually negligible. On the other hand, to account for strong colloid-ion and colloid-colloid interactions require a full nonlinear theory. One approach that has proven to be very useful for describing the nonlinear correlations between the colloidal particles and the counterions is the concept of charge renormalization. ${ }^{1,18,19}$ The idea is that strong electrostatic attraction between the colloidal particles and their counterions will lead to accumulation of counterions near the colloidal surface. These counterions can be considered to be "condensed" (strongly bound) to the colloidal particle, effectively renormalizing its bare charge. For strongly charged colloidal particles the renormalized charge will, in general, be much smaller in magnitude than the bare charge. ${ }^{18}$

An alternative, but equivalent way of modeling colloidal suspensions, is to explicitly trace out the microion degrees of freedom in a semi-grand-canonical partition function. ${ }^{20}$ This way the multi-component colloidal suspension is mapped onto an equivalent one-component system in which only the colloidal particles are explicit. This coarse-graining procedure defines the, so-called, one component model (OCM). In this approach, all the contributions coming from the traced-out microions are implicit in the effective interactions between the colloidal particles. ${ }^{20}$ The apparent simplification over the original problem is only formal, since the effective interaction between the colloidal particles now has a many-body character $^{20-22}$ and is state-dependent, ${ }^{20,21}$ further complicating the thermodynamic calculations..$^{23-26}$

For weakly charged colloidal particles, the effective interaction potential in the OCM takes a particularly simple form known as the Derjaguin-Landau-Overbeek-Verwey (DLVO) pair potential, ${ }^{27,28}$

$$
\beta u(r)=\lambda_{B}\left(\frac{Z e^{\kappa a}}{1+\kappa a}\right)^{2} \frac{e^{-\kappa r}}{r},
$$

where $a$ and $-Z q$ are the colloidal radius and charge, respectively. The inverse Debye screening length is $\kappa$ $\equiv \sqrt{4 \pi \lambda_{B}\left(\rho_{+}+\rho_{-}\right)}$, where $\rho_{+}$and $\rho_{-}$are the mean concentrations of the monovalent counterions and coions inside the suspension, and $\lambda_{B} \equiv \beta q^{2} / \epsilon$ is the Bjerrum length. Due to the global charge neutrality, $\rho_{+}-\rho_{-}-Z \rho=0$, where $\rho$ is the concentration of colloidal particles. 
For strongly charged colloidal particles, the linear DLVO theory is not sufficient to describe the pairwise interactions. The nonlinear effects, however, can partially be included into DLVO potential by explicitly accounting for the counterion condensation. This can be achieved by replacing the bare colloidal charge in Eq. (1) by the renormalized effective charge $Z \rightarrow Z_{\text {eff. }}$ The charge renormalization accounts for the strong nonlinear particle-counterion correlations near the colloidal surfaces.

The formal trace over the microion degrees of freedom in the partition function results in many-body effective OCM interactions. ${ }^{21}$ In particular, besides the DLVO pair potential and higher multi-body potentials, there also appear zerothorder terms which do not depend on the specific colloidal coordinates, but only on the average colloidal and ionic concentrations. These are known as the "volume terms." "3,9 Since the volume terms do not depend on the colloidal coordinates, they have no influence on the structural properties of the OCM. On the other hand, they appear to be relevant for calculating the thermodynamic functions, and in particular the equation of state of the colloidal suspension..$^{20,25,26}$ In general, the effective OCM interactions between the colloidal particles depend on the particular colloidal configuration. This point has been demonstrated in PM simulations where it was found that bcc and fcc arrangements lead to different effective pair forces between the colloidal particles. ${ }^{29}$

The question of whether the effective potential models based purely on pair interactions are sufficient to study the thermodynamics of a fully multi-component system is still under discussion. ${ }^{23,24}$ In the case of charged colloidal systems, the problem is even more subtle, since such systems must obey additional constraints, i.e., global electro-neutrality and the well-known Stillinger-Lovett moment conditions. ${ }^{30}$ As a consequence, many theoretical tools originally designed for unconstrained systems have to be reformulated before they can be applied to charged systems. ${ }^{31,32}$

The aim of this work is to address some thermodynamic inconsistencies which arise when different routes are used to calculate the thermodynamic functions of charged colloidal suspensions. To this end, we will use the renormalized Jellium model (RJM), from which both the renormalized charge and the osmotic pressure can be easily calculated. ${ }^{33,34}$ From the renormalized charge, the effective pair potential-and hence the colloid-colloid pair correlation functions-can be obtained using the OCM Ornstein-Zernike (OZ) equation ${ }^{35}$ with an appropriate closure. Knowing the correlations, it is possible to calculate the osmotic compressibility using the Kirkwood-Buff (KB) fluctuation theory. ${ }^{36}$ In this work we will compare the osmotic compressibilities of the RJM calculated using both the contact theorem and the KB fluctuation relations.

The paper is organized as follows. In Sec. II we will briefly review the theoretical methods used for the thermodynamic investigations - the RJM, the Donnan equilibrium, and the Kirkwood-Buff relations. In Sec. III, we will briefly discuss the simulation techniques employed in this study. The results will be presented in Sec. IV, and conclusions, discussion, and suggestions for the future investigations will be given in Sec. V.

\section{THEORETICAL BACKGROUND}

\section{A. The renormalized Jellium model}

The RJM is a model that allows one to calculate the effective charge of colloidal particles and the thermodynamic properties of colloidal suspensions based on the mean-field Poisson-Boltzmann-like (PB) equation. RJM is known to be very accurate for salt-free colloidal suspensions with monovalent counterions. In contrast to the traditional cell model (CM), where a lattice-like structure is assumed for colloidal particles, in the RJM the colloidal correlations are modeled by a uniform neutralizing background. The major conceptual advantage of the RJM over the CM is that the DLVO pair potential Eq. (1) is exact within the RJM formalism, while for CM there is no pairwise interaction between the colloidal particles. ${ }^{33}$ Thus, the effective charges calculated using CM have no clear connection with the DLVO potential. Recently, the RJM was successfully extended to incorporate inter-colloidal correlations, ${ }^{37,38}$ the multivalent counterions, ${ }^{39}$ and colloidal polydispersity. ${ }^{40}$

In the RJM, one colloidal particle of charge $-Z_{\text {bare }} q$ and radius $a$ is fixed at the origin of the coordinate system. The distribution of free (uncondensed) ions around this particle is assumed to follow the Boltzmann distribution, $\rho_{ \pm}(r)$ $=\rho_{ \pm} e^{\mp \beta q \psi(r)}$, where $\rho_{ \pm}$are the counterion and coion mean densities, and $\psi(r)$ is the mean electrostatic potential. The remaining colloidal particles, along with their condensed counterions, are taken to provide a uniform neutralizing background of charge density $-Z_{\text {eff }} q \rho$. The reduced mean electrostatic potential $\phi(r)=\beta q \psi(r)$ then satisfies the Poisson-Boltzmann-Jellium (PBJ) equation,

$$
\nabla^{2} \phi(r)=-4 \pi \lambda_{B}\left(\rho_{+} e^{-\phi(r)}-\rho_{-} e^{\phi(r)}-Z_{e f f} \rho\right) .
$$

This equation can be numerically solved with the boundary conditions $\phi(r \rightarrow \infty) \rightarrow 0$ and $\left.\frac{d \phi(r)}{d r}\right|_{r=a}=\frac{Z_{\text {bar }} \lambda_{B}}{a^{2}}$. The first condition defines the zero of the electrostatic potential in the bulk of suspension, while the second one determines the electric field at the colloidal surface using the Gauss law.

Far from the central colloidal particle - the region where the electrostatic potential is weak-the PBJ equation can be linearized, resulting in the following long-distance behavior:

$$
\phi(r)=-\frac{Z_{e f f} \lambda_{B} e^{\kappa a}}{(1+\kappa a)} \frac{e^{-\kappa r}}{r},
$$

where $\left.\kappa=\sqrt{4 \pi \lambda_{B}\left(\rho_{+}+\rho_{-}\right)}=\sqrt{4 \pi \lambda_{B}\left(2 \rho_{-}+Z_{\text {eff }}\right.} \rho\right)$ is the effective screening length, and where we have used the global charge neutrality condition $\rho_{+}-\rho_{-}-\rho Z_{\text {eff }}=0$. Note that in the far-field, the bare charge $Z_{\text {bare }}$ is replaced by the renormalized charge $Z_{\text {eff }}$, reflecting the nonlinear colloidcounterion correlations at the colloidal surface.

For a given salt and colloidal concentrations, $\rho_{-}$and $\rho$, respectively, the effective charge is calculated by matching the numerical solution of Eq. (2) with the linearized potential Eq. (3), in the far-field. Since within the RJM the background charge arises from the smeared-out charge of colloidal particles and their condensed counterions, the self-consistency requires that the effective colloidal charge must be the same as the charge of the uniform neutralizing background. This procedure can be easily implemented numerically. ${ }^{41}$ Suppose 
that we know $Z_{\text {eff }}$, then from Eq. (3) we will also know the potential and the electric field in the far-field region. We can then integrate the PBJ equation using a standard Rounge-Kutta algorithm to obtain the electrostatic potential all the way up to the colloidal surface. The corresponding bare colloidal charge $Z_{\text {bare }}$ is obtained using the Gauss law at the colloidal surface. In reality, of course, one wants to calculate the effective charge for a given bare charge. This can be done by varying $Z_{\text {eff }}$ until the desired $Z_{\text {bare }}$ is found. In practice, this can be easily implemented numerically by coupling a Newton-Raphson root-finding subroutine to the PBJ solver.

The osmotic pressure within the RJM is given by

$$
\beta P=\rho_{+}+\rho_{-},
$$

where $\rho_{ \pm}$are the bulk concentrations of free coions and counterions. In spite of its apparent simplicity, this ideal-gas-like equation of state requires a knowledge of microion concentrations in the far-field which, in turn, depend on the charge renormalization and osmotic equilibrium with the salt reservoir. We should also note that unlike for CM, for which the contact value theorem is exact, ${ }^{42-45} \mathrm{Eq}$. (4) of the RJM is only valid in the mean-field approximation. We will later argue that the failure to properly account for ionic correlations leads to the thermodynamics inconsistencies in the RJM.

\section{B. The Donnan equilibrium}

In this work, we will consider a colloidal suspension in contact with a salt reservoir. The system is separated from the reservoir by a semi-permeable membrane which allows for a free flux of microions. The ionic concentration inside the suspension will then be determined by the osmotic equilibrium with the salt reservoir. Contrary to uncharged systems, for which the osmotic equilibrium simply results in a solvent flow from a solute poor to a solute rich region, the osmotic equilibrium in charged systems is also constrained by the overall charge neutrality of the system. Physically, this is reflected in the appearance of a potential difference across the semipermeable membrane which controls the overall build up of charge in the system. ${ }^{16,17}$ This potential difference is known as the Donnan potential. ${ }^{46}$ From a theoretical point of view, it can also be thought of as a Lagrange multiplier used to enforce the charge neutrality of the system. ${ }^{16,47}$

In equilibrium, the ionic electrochemical potentials inside the system must be equal to the ones in the salt reservoir. Neglecting the electrostatic correlations between the microions, the ionic concentrations in the bulk and reservoir are related by $\rho_{ \pm}=\rho_{s} e^{\mp \phi_{D}}$, where $\rho_{s}$ is the salt concentration in the reservoir, and $\phi_{D}$ is the adimensional Donnan potential. Using the charge neutrality condition for free ions, $\rho_{+}-\rho_{-}-\rho Z_{\text {eff }}=0$, the Donnan potential can be eliminated to yield the bulk concentrations of free (uncondensed) microions,

$$
\rho_{ \pm}=\frac{1}{2}\left(\sqrt{\left(\rho Z_{e f f}\right)^{2}+\left(2 \rho_{s}\right)^{2}} \pm Z_{e f f} \rho\right)
$$

This expression can be used, together with the equation of state Eq. (4), to write the osmotic pressure $\beta \Pi$ as,

$$
\beta \Pi \equiv \beta P-2 \rho_{s}=\rho+\sqrt{\left(\rho Z_{e f f}\right)^{2}+\left(2 \rho_{s}\right)^{2}}-2 \rho_{s},
$$

where we have also added the colloidal ideal gas contribution $\beta P_{c}=\rho$. It is important to stress that the above expression for the osmotic pressure completely ignore the microion correlations. This can be justified as long as the concentration of coions in the bulk is very low. The colloid-counterion correlations are taken into account through the colloidal charge renormalization.

Using Eq. (6), two important limits can be verified. For high-salt concentrations $-Z_{\text {eff }} \rho / 2 \rho_{s} \ll 1$, salt-dominated regime - there is no significant variation in the ionic concentrations across the membrane and the osmotic pressure (6) is small. On the other hand, in the limit $Z_{\text {eff }} \rho / 2 \rho_{s} \gg 1$ - the counterion-dominated regime - there is a significant variation in the microion concentration between the bulk suspension and the reservoir and the osmotic pressure is large. ${ }^{16}$

The inverse osmotic compressibility $\chi_{T}^{-1}=\rho\left(\frac{\partial \Pi}{\partial \rho}\right)_{\rho_{s}, T}$ follows directly from Eq. (6),

$$
\beta \chi_{T}^{-1}=\rho+\frac{\rho^{2} Z_{e f f}^{2}}{\sqrt{\left(\rho Z_{e f f}\right)^{2}+\left(2 \rho_{s}\right)^{2}}}\left(1+\frac{d \log \left(Z_{e f f}\right)}{d \log (\eta)}\right),
$$

where $\eta=4 \pi a^{3} \rho / 3$ is the colloidal volume fraction. The derivative on the right-hand side of this expression can be neglected, since in the RJM the effective charge depends only weakly on the colloidal volume fraction. ${ }^{33,34}$

\section{The Kirkwood-Buff relation}

Once the nonlinear colloid-ion correlations are properly taken into account through the charge renormalization, the DLVO pair potential Eq. (1) can be used to investigate the structural properties of the suspension. This can be done by solving the OCM Ornstein-Zernike equation

$$
h(\mathbf{r})=c(\mathbf{r})+\rho \int h\left(\mathbf{r}^{\prime}\right) c\left(\left|\mathbf{r}-\mathbf{r}^{\prime}\right|\right) d \mathbf{r}^{\prime},
$$

where $h(\mathbf{r})$ and $c(\mathbf{r})$ are the total and the direct correlation functions, respectively. This equation has to be supplemented by an appropriate closure relation between $h(\mathbf{r})$ and $c(\mathbf{r}){ }^{35}$

Once the structural properties are known, the thermodynamic informations can then be obtained using the KB fluctuation theory. ${ }^{36} \mathrm{~KB}$ theory allows us to express the thermodynamic functions, such as the osmotic coefficients and the compressibilities, as integrals over the pair correlation functions. Originally formulated for unconstrained mixtures, KB theory requires some extra care when extended to systems in which the number densities of different components are not independent. $^{23,31,32}$ This is precisely the case for the charged systems, for which long-range Coulomb interaction requires an overall charge neutrality. In addition to this, there are also other constraints known as the Stillinger-Lovett moment conditions, that restrict the fluctuations of different components ${ }^{30}$ of a charged system. A naive application of the original KB theory to charged systems leads to undetermined results. ${ }^{35,48}$ 
One way of avoiding these difficulties is to study the KB integrals for arbitrary $k$ vectors in the Fourier space, ${ }^{32}$ taking the limit $k \rightarrow 0$ at the end of the calculations. The extended KB theory then relates the osmotic compressibility with the Fourier transform of the total correlation function $\hat{h}(\mathbf{k})$,

$$
\chi_{T}=1+\rho \int h(\mathbf{r}) d \mathbf{r}=1+\rho \hat{h}(0)
$$

Using OZ equation, this expression can be inverted to yield

$$
\left(\frac{\partial \beta P}{\partial \rho}\right)_{\rho_{s}, T}=1-\rho \hat{c}(0) \text {. }
$$

KB theory shows that the knowledge of colloidal pair correlation function is sufficient for calculating the equation of state of the colloidal suspension. Curiously, Eqs. (9) and (10) rely only on the pair correlations which are well modeled using the effective pair potential, Eq. (1). This suggests that the the zero-order volume terms, which depend on the colloidal concentration, ${ }^{3,9}$ are already accounted for by colloidal pair correlations.

\section{MONTE CARLO SIMULATIONS}

To explore the validity of the RJM model, we perform Monte Carlo simulations to obtain the "exact" pairwise interaction potential. The simulations are performed for several fixed distances $R$ between two spherical colloidal particles of charge $-Z_{\text {bare }} q$, which are restricted to move along the main diagonal of a box of side length $L=180 \AA$ A. Colloid particle 1 is located at $x, y, z=-R / 2 \sqrt{3}$, and colloidal particle 2 at $x, y, z=R / 2 \sqrt{3}$. In order to keep the electro-neutrality, $2 Z_{\text {bare }}$ microions of charge $q$ are also present in the simulation box. If salt is added to the system, then $L^{3} \rho_{S}$ microions of charge $q$ and $L^{3} \rho_{S}$ microions of charge $-q$ are included inside the box. The total number of microions in the system is then $N=2 L^{3} \rho_{S}+2 Z_{\text {bare }}$. The radii of all the ions are set to 2 $\AA$. The usual Coulomb potential is considered between all the charged species. The total energy used in the MC simulations is,

$$
\frac{\beta}{\lambda_{B}} E=\sum_{i=1}^{N-1} \sum_{j=i+1}^{N} \frac{z_{i}^{2}}{r_{i j}}-\sum_{i=1}^{N} \frac{Z_{\text {bare }} z_{i}}{r_{\overline{1} i}}-\sum_{i=1}^{N} \frac{Z_{\text {bare }} z_{i}}{r_{\overline{2} i}},
$$

where $z_{i}$ is the charge valence of the ion $i(+1$ or -1$), r_{i j}$ is the distance between two ions $i$ and $j, r_{\overline{1} i}$ and $r_{\overline{2}}$ are the distances between the ion $i$ and the colloidal particles 1 and 2, respectively. Since we consider periodic boundary conditions, the Ewald summation technique is employed. ${ }^{49}$ The equilibration is achieved after $2.5 \times 10^{3}$ simulation steps per particle; every 100 movements per particle an uncorrelated state is saved. The mean force is calculated using $1 \times 10^{4}$ uncorrelated configurations.

The average electrostatic force on a colloidal particle (positive force corresponds to repulsion), along the diagonal direction is

$$
\bar{F}_{e}(R)=\left\langle\sum_{i=1}^{N} \frac{Z_{\text {bare }} Z_{i}}{2}\left(\frac{\cos \theta_{\overline{1} i}}{r_{\overline{1} i}^{2}}+\frac{\cos \theta_{\overline{2} i}}{r_{\overline{2} i}^{2}}\right)\right\rangle+\frac{Z_{\text {bare }}^{2}}{R^{2}},
$$

where $\bar{F}_{e}(R)=\frac{\beta}{\lambda_{B}} F_{e}(R), \theta_{\overline{1} i}$ and $\theta_{\overline{2} i}$ are the angles between the diagonal and the line connecting the particle $i$ to the colloid 1 and the colloid 2, respectively. These distances are measured from the diagonal in the counterclockwise, for particle 1 , and in the clockwise, for particle 2 direction, respectively. The Ewald technique is used to calculate the electrostatic forces. Besides the average electrostatic force, there is also an entropic depletion force which must be taken into account. To do this we use the method of Wu et al. ${ }^{50}$ which consists of a small displacement of the colloidal particles along the diagonal (while the microions remain in a fixed configuration) in order to count the resulting overlaps between the colloidal particle and the microions. This entropic force can be expressed as

$$
\bar{F}_{d}(R)=\frac{\left\langle N_{1}^{c}\right\rangle-\left\langle N_{1}^{f}\right\rangle+\left\langle N_{2}^{c}\right\rangle-\left\langle N_{2}^{f}\right\rangle}{2 \Delta R \lambda_{B}},
$$

where $\bar{F}_{d}(R)=\frac{\beta}{\lambda_{B}} F_{d}(R), N_{1}^{c}$ is the number of overlaps of colloidal particle 1 with the microions (both anions and cation), after a small displacement $\Delta R(\approx 1 \AA)$ in the direction of the colloidal particle 2 (superscript c stands for closer) and $N_{1}^{f}$, is the number of overlaps after a displacement $\Delta R$ in the opposite direction (superscript f stands for farther). Similarly $N_{2}^{c}$ and $N_{2}^{f}$, are the number of overlaps of colloidal particle 2 with the microions after a displacement $\Delta R$ in the direction of the colloidal particle 1 and in the opposite direction, respectively. The effective pair potentials can then be calculated by integrating the mean force, $-\lambda_{B} \int_{R_{\max }}^{R} d R^{\prime}\left[\bar{F}_{e}\left(R^{\prime}\right)+\bar{F}_{d}\left(R^{\prime}\right)\right]$, where $R_{\max }$ is the reference distance at which the interaction between the two colloidal particles is negligible.

\section{RESULTS}

We are now in a position to compare the thermodynamic predictions from Eqs. (7) and (9). To this end, the OZ equation is numerically solved using the hypernetted-chain (HNC) closure

$$
c(\mathbf{r})=h(\mathbf{r})-\log (h(\mathbf{r})+1)-\beta u(\mathbf{r}) .
$$

This closure is known to be very accurate for the Yukawa-like pair potentials. ${ }^{35,51}$ For a given reservoir salt concentration $\rho_{s}$ and volume fraction $\eta$, the pair potential is given by (1), with the effective charge calculated using the RJM.

In order to test the accuracy of the effective pair potential predicted by the RJM, in Fig. 1 we compare it with the results of the Monte Carlo simulations. As can be seen, the DLVO pair potential with the bare colloidal charge considerably overestimate the effective colloid-colloid interaction. On the other hand, the pair-potential predicted by the RJM agrees well with the MC simulations. For short separations, however, a small deviation from the Yukawa functional form is evident. This nonlinear screening interaction is a consequence of neglected correlations among the counterions sandwiched between the colloidal surfaces.

In Fig. 2, the colloid-colloid pair correlation function $g(r)$ calculated using the RJM and the HNC integral equation, is compared with the results of the MC simulations ${ }^{52}$ in the 

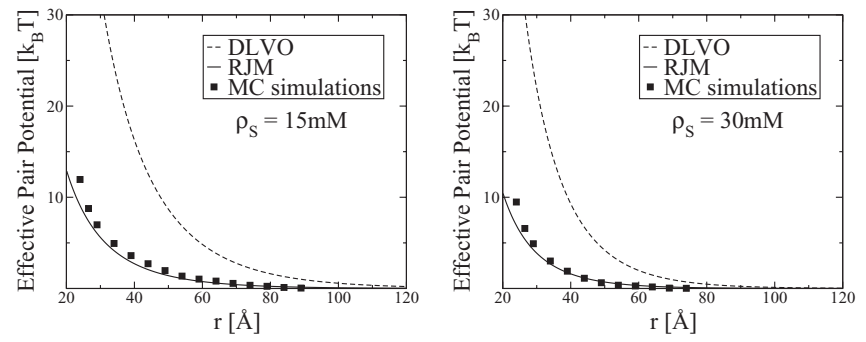

FIG. 1. Effective pair potentials calculated using the MC simulations (squares), bare DLVO pair potential (dashed line), and DLVO with RJM effective parameters (solid line) for $Z_{\text {bare }}=20, a=10 \AA$, and $\lambda_{B}=7.2 \AA$.

high-salt concentration regime. Again, we see a good agreement between the theory and the simulations.

Figures 1 and 2 show that the effective charges calculated using the RJM are able to correctly predict both the pair interactions and the structural properties of colloidal suspensions containing added electrolyte. We next check if this good agreement also extends to the thermodynamic functions. Unfortunately, very quickly we run into difficulties. We find that for the intermediate salt concentrations, the osmotic compressibility calculated using the $\mathrm{KB}$ fluctuation relation Eq. (9) strongly deviates from the one calculated using the RJM equation of state (JEOS), Eq. (6). The discrepancy between the two routes can be clearly seen in Fig. 3, which shows the osmotic compressibility $\chi_{o s m}$ as a function of the reservoir salt concentration $\rho_{s}$, for colloidal particles of bare charge $Z=1000$ and various volume fractions. Although both routes agree in the high-salt regime, there are strong deviations at intermediate salt concentrations. Furthermore, as the colloidal concentration increases, the discrepancy between the two thermodynamic routes becomes stronger. At low volume fractions and high-salt concentration, both routes approach the correct ideal gas limit $\chi_{\text {osm }} \approx 1$, when strong screening makes the system behave as a dilute suspension of hard spheres.

The question that arises then is: Which thermodynamic route is more reliable? Unfortunately, the answer is not very clear. Due to the difficulty of performing large scale simulations on suspensions containing electrolyte, there are very little data available to us to answer this question. Furthermore, there is also a scarcity of the experimental data dealing with osmotic properties of charged colloidal suspensions. In Fig. 4, we compare both the osmotic pressure calculated using the JEOS and the KB fluctuation theory with the
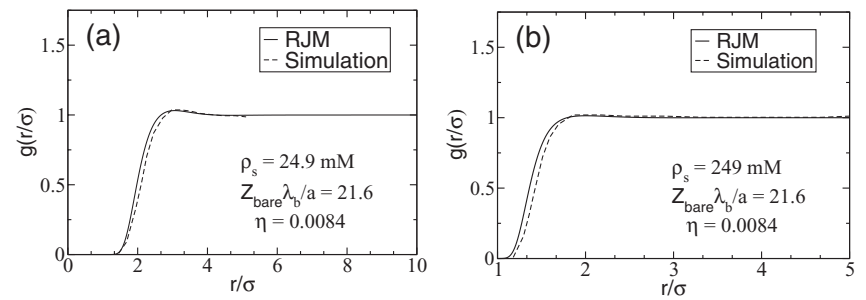

FIG. 2. Colloid-colloid pair correlation functions obtained using the MC simulations (Ref. 52) and the RJM-OZ approach, for (a) $\rho_{s}=24.9 \mathrm{mM}$ and (b) $\rho_{s}=249 \mathrm{mM}$. In both cases, the bare charge is $Z_{\text {bare }} \lambda_{B} / a=21.6$, and the volume fraction is $\eta=0.0084$.
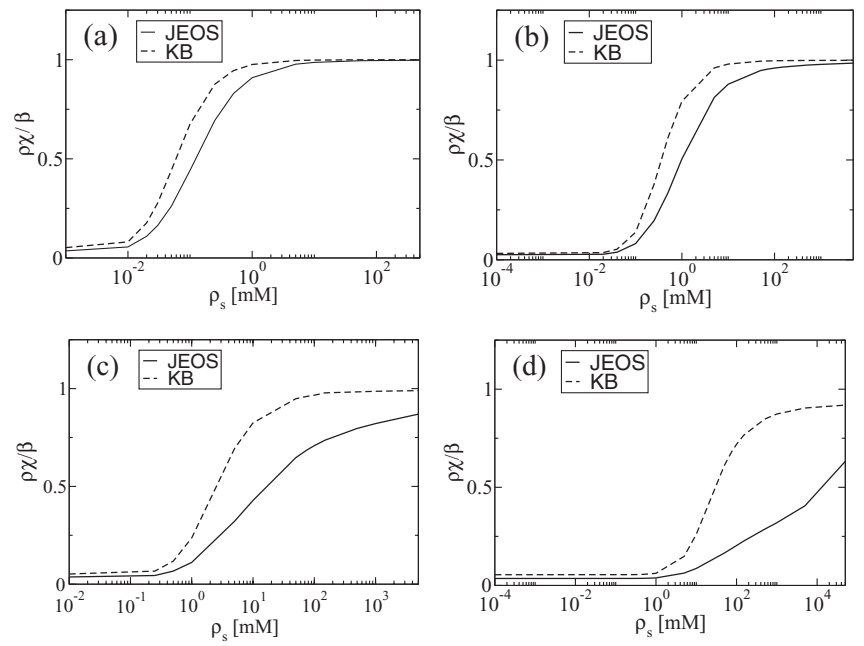

FIG. 3. Reduced osmotic compressibility $\tilde{\chi} \equiv \rho \chi / \beta$ as a function of the reservoir salt concentration $\rho_{s}$ for colloidal particles of radius $a=30 \AA$ and bare charge $Z=1000$. The colloidal volume fractions are: (a) $\eta=10^{-5}$, (b) $\eta=10^{-4}$, (c) $\eta=10^{-3}$, and (d) $\eta=10^{-2}$. We see a dramatic discrepancy between the predictions of the JEOS (solid lines) and the KirkwoodBuff fluctuation theory (dashed lines), especially at intermediate salt concentrations and high volume fractions.

experimental measurements of Rasa et al. ${ }^{53}$ Neither one of the thermodynamic routes seems to be able to accurately describe this experimental data. Most likely this is a consequence of the strong electrostatic correlations between the ions resulting from the use of a low dielectric solvent by Rasa et al. Nevertheless, the fluctuation route seems to give results in a closer agreement with the experimental data than the JEOS. This suggest that for the RJM the fluctuation route might be more reliable for calculating the thermodynamic functions. We will now explore the possible causes of the discrepancy between the two thermodynamic routes.

\section{A. Colloid-colloid correlations}

One possibility is that the discrepancy observed in Fig. 3 is due to the way that colloidal correlations enter into the theory in the two thermodynamic routes. ${ }^{54,55} \mathrm{In}$ deed, while the colloid-colloid correlations are neglected in the JEOS, they contribute to the osmotic pressure calculated using the KB formalism Eq. (9), since the $\mathrm{HNC}$ equation ${ }^{54}$ used to obtain the correlation function takes into account
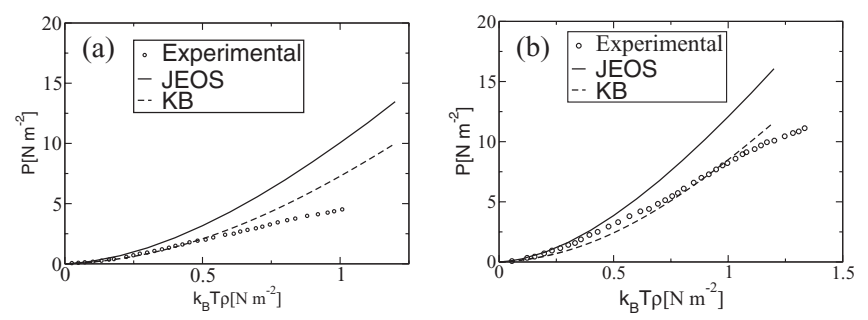

FIG. 4. Comparison between the osmotic pressure calculated using the JEOS, (solid line) and the explicit integration of Eq. (9) (dashed line), with the experimental results reported in Ref. 53. The reservoir salt concentration is $\rho_{s}=8 \mu \mathrm{M}$, while the Bjerrum length is $\lambda_{B}=2.38 \mathrm{~nm}$, the colloidal radius is $a=21.9 \mathrm{~nm}$, and colloidal charges are: (a) $Z=34$ and (b) $Z=40$. 
the colloidal hard-cores. The colloid-colloid repulsion is particularly important for large volume fractions and high-salt concentration $\left(Z \rho / 2 \rho_{s} \ll 1\right)$, when ionic contribution to the osmotic pressure is small. To asses the relevance of these correlations, we can add to the JEOS the excess colloidal virial pressure,

$$
\beta P^{e x}=-\frac{2 \pi \rho^{2}}{3} \int_{0}^{\infty} g(r) r^{3} \frac{d \beta u}{d r} d r+2 \pi \rho^{2} V \int_{0}^{\infty} g(r) \frac{\partial \beta u}{\partial V} r^{2} d r,
$$

where $u(r)$ is the effective pair potential in the OCM description. The first term on the right-hand side of this equation represents the standard excess virial pressure for the onecomponent system, while the second term accounts for the density dependent effective pair potential. This term is essential to reproduce the correct Debye-Hückel limiting law in the infinite dilution limit. ${ }^{56}$ Substitution of Eq. (1) into Eq. (15) produces the following expression for the excess pressure,

$$
\begin{aligned}
\beta P^{e x}= & \frac{2 \pi \rho^{2}}{3} \int_{0}^{\infty} g(r) \beta u(r)(\kappa r+1) r^{2} d r \\
& -\pi \rho^{2} \int_{0}^{\infty} g(r) \beta u(r)\left(\kappa r-\frac{2(\kappa a)^{2}}{1+\kappa a}\right) r^{2} d r,
\end{aligned}
$$

where $u(r)$ is the effective colloidal pair potential, Eq. (1). For all the parameters studied here, however, we find that $\left|P^{e x}\right|$ $\ll P_{\text {Jell }}$, and the effect of colloidal correlations is too small to account for the strong discrepancy observed in Fig. 3.

\section{B. Ion-ion correlations}

As the salt concentration increases, the mean distance between the cations and anions becomes small, leading to strong inter-ionic correlations. Such correlations are completely ignored by the mean-field Poisson-Boltzmann equation, which is the basis of the RJM. Indeed, in the absence coions (and for monovalent counterions), the RJM model was found to provide an excellent account of both thermodynamic and structural properties of charged suspensions. ${ }^{33,34,37,38}$ This good accuracy of the model is the result of large characteristic distance between the counterions inside a salt-free suspension. On the other hand, presence of salt leads to strong cationanion correlations neglected in the RJM.

In order to explore the influence of inter-ionic correlations on the osmotic pressure in a colloidal suspension, we modify the JEOS by adding the correlational Debye-Hückel contribution, ${ }^{1,57}$

$$
\beta P^{e x}=-\frac{\kappa^{3}}{24 \pi} .
$$

Figure 5 shows the osmotic compressibilities resulting from addition of Eq. (17) to the JEOS, Eq. (6). As can be seen from this figure, incorporation of ionic correlations even at this leading-order level, significantly improves the agreement between the two thermodynamic routes, especially at large colloidal volume fractions. This simple calculation suggests that the thermodynamic consistency of the RJM can be restored by incorporating the inter-ionic correlations into the RJM. Unfortunately, at the moment, it is not clear how the inter-ionic correlations can be included into the RJM in a
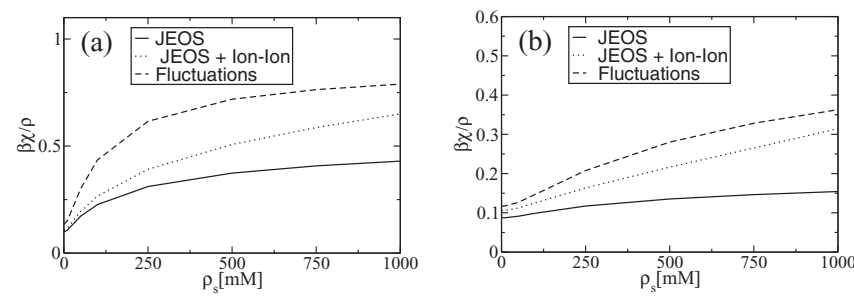

FIG. 5. Comparison between the osmotic compressibilities calculated using the JEOS Eq. (6) (solid lines); the JEOS with explicit ionic correlations Eqs. (6) and (17) (point lines); and the KB fluctuation theory, Eq. (9) (dashed lines). The radius of colloidal particles is $a=$ $10 \AA$, the bare colloidal charge is $Z=1000$, and the Bjerrum length is $\lambda_{b}=7.2 \AA$. The volume fractions are: (a) $\eta=0.01$ and (b) $\eta=0.05$.

fully self-consistent fashion. This will be the subject of future research.

\section{SUMMARY AND CONCLUSIONS}

We have reported an inconsistency arising when different routes are employed to calculate the thermodynamic functions in the the RJM. The discrepancies are particularly strong at moderate salt concentrations. On the other hand, comparing the predictions of the RJM with the MC simulations, we see that the model accurately accounts for the effective pair interactions and the colloidal correlation functions, even for suspensions containing electrolyte.

Thermodynamic inconsistency between different routes is not particular to the RJM and is found for many other systems. ${ }^{58}$ Even for a Debye-Hückel electrolyte, the osmotic compressibility calculated via the PM virial equation is quite different from the predictions of the fluctuation theory. ${ }^{3}$ In these cases, $\mathrm{MC}$ simulations are particularly helpful to choose the more accurate route to thermodynamics. ${ }^{58}$ Unfortunately, simulations of charged colloidal suspensions at even moderate salt concentrations are still too computationally demanding while the experimental data is still very scarce. The experimental and the simulational data available to us seems to indicate that KB fluctuation relations provide a more reliable route to thermodynamics of the RJM. The KB route seems to partially account for the inter-ionic correlations which are completely neglected by the JEOS. Although, these correlations are negligible in the absence of coions they, however, become relevant when salt concentration increases and the characteristic distance between the cations and the anions becomes small. ${ }^{1}$ Unlike for salt-free suspensions, for which the electrostatic correlations are important only when ions are multivalent, for suspensions with salt ionic correlations are important even with monovalent ions when the concentration of salt is larger than few tens of $\mathrm{mM}$.

It is well known that ionic correlations have a strong influence on charged colloidal suspensions. For example, the exclusion volume effects can strongly modify the ionic adsorption near the colloidal surface, ${ }^{60}$ while high size asymmetry between coions and counterions may lead to colloidal charge reversal even with monovalent microions. ${ }^{59}$ Furthermore, it has been recently demonstrated by both molecular dynamic simulations and the PM Ornstein-Zernike equations 
that deviations from the DLVO theory can be observed in the region of high-salt concentrations. ${ }^{61}$ None of these effects are captured by the mean-field PB theory, and demand more elaborated approaches which properly account for the ionic correlations. In Sec. IV B, we showed that even a simple incorporation of the DH contribution into the osmotic pressure already brings the JEOS and the fluctuation results into a closer agreement. A fully self-consistent incorporation of ionic correlations into the RJM requires, however, development of a new methodology closer in spirit to the density functional theory.

\section{ACKNOWLEDGMENTS}

This work was partially supported by the CNPq, FAPERGS, INCT-FCx, and by the US-AFOSR under the Grant No. FA9550-09-1-0283.

${ }^{1}$ Y. Levin, Rep. Prog. Phys. 65, 1577 (2002).

${ }^{2}$ R. Messina, J. Phys.: Condens. Matter 21, 113102 (2009).

${ }^{3}$ L. Belloni, J. Phys.: Condens. Matter 12, R549 (2000).

${ }^{4}$ K. Mahdi and M. O. de la Cruz, Macromolecules 33, 7649 (2000).

${ }^{5}$ A. Diehl, M. C. Barbosa, and Y. Levin, Europhys. Lett. 53, 86 (2001).

${ }^{6}$ Y. Levin, E. Trizac, and L. Bocquet, J. Phys.: Condens. Matter. 15, S3523 (2003).

${ }^{7}$ A. R. Denton, Phys. Rev. E 76, 051401 (2007).

${ }^{8}$ B. Lu and A. R. Denton, Phys. Rev. E 75, 061403 (2007).

${ }^{9}$ J.-P. Hansen and H. Löwen, Annu. Rev. Phys. Chem. 51, 209 (2000).

${ }^{10}$ G. Vernizzi, G. I. Guerrero-Garcia, and M. O. de la Cruz, Phys. Rev. E 84, 016707 (2011).

${ }^{11}$ A. P. dos Santos and Y. Levin, Phys. Rev. Lett. 106, 167801 (2011).

${ }^{12}$ A. P. dos Santos, A. Bakhshandeh, and Y. Levin, J. Chem. Phys. 135, 044124 (2011).

${ }^{13}$ A. Bakhshandeh, A. P. dos Santos, and Y. Levin, Phys. Rev. Lett. 107, 107801 (2011).

${ }^{14}$ L. Lue and P. Linse, J. Chem. Phys. 135, 224508 (2011).

${ }^{15}$ M. M. Hatlo, R. van Roij, and L. Lue, Europhys. Lett. 97, 28010 (2012).

${ }^{16}$ M. Tamashiro, Y. Levin, and M. Barbosa, Eur. Phys. J. B 1, 337 (1998).

${ }^{17}$ M. Deserno and H. H. von Grünberg, Phys. Rev. E 66, 011401 (2002).

${ }^{18}$ L. Belloni, Colloids Surf., A 140, 227 (1998).

${ }^{19}$ P. Gonzalez-Mozuleos and M. O. de la Cruz, Physica A 387, 5362 (2008).

${ }^{20}$ C. N. Likos, Phys. Rep. 348, 247 (2001).

${ }^{21}$ A. R. Denton, in Nanostructured Soft Matter: Experiment, Theory, Simulation and Perspectives, edited by A. V. Zvelindovsky (Springer, Dordrecht, 2007).

${ }^{22}$ A. R. Denton, Phys. Rev. E 67, 011804 (2003).

${ }^{23}$ F. H. Stillinger, H. Sakai, and S. Torquato, J. Chem. Phys. 117, 288 (2002).

${ }^{24}$ A. A. Louis, J. Phys.: Condens. Matter 14, 9187 (2002).

${ }^{25}$ M. Dijkstra, R. van Roij, and R. Evans, J. Chem. Phys. 113, 4799 (2000).
${ }^{26}$ E. Trizac et al., Phys. Rev. E 75, 011401 (2007).

${ }^{27}$ B. V. Derjaguin and L. D. Landau, Acta Physicochim. URSS 14, 633 (1941).

${ }^{28}$ E. J. Verwey and J. T. G. Overbeek, Theory of the Stability of Lyophobic Colloids (Elsevier, Amsterdam, 1948).

${ }^{29}$ J. Dobnikar, Y. Chen, R. Rzehak, and H. H. von Grünberg, J. Chem. Phys. 119, 4971 (2003).

${ }^{30}$ F. Stillinger and R. Lovett, J. Chem. Phys. 49, 1991 (1968).

${ }^{31}$ H. L. Friedman and P. S. Ramanathan, J. Phys. Chem. 74, 3756 (1970).

${ }^{32}$ P. G. Kusalik and G. N. Patey, J. Chem. Phys. 86, 5110 (1987).

${ }^{33}$ E. Trizac and Y. Levin, Phys. Rev. E 69, 031403 (2004).

${ }^{34}$ S. Pianegonda, E. Trizac, and Y. Levin, J. Chem. Phys. 126, 014702 (2007).

${ }^{35}$ J.-P Hansen and I. R. McDonald, Theory os Simple Liquids (Academic, New York, 1976).

${ }^{36}$ J. G. Kirkwood and F. P. Buff, J. Chem. Phys. 19, 774 (1951).

${ }^{37}$ R. Castaneda-Priego, L. F. Rojas-Ochoa, V. Lobaskin, and J. C. MixtecoSanchez, Phys. Rev. E 74, 051408 (2006).

${ }^{38}$ T. E. Colla, Y. Levin, and E. Trizac, J. Chem. Phys. 131, 074115 (2009).

${ }^{39}$ T. E. Colla and Y. Levin, J. Chem. Phys. 133, 234105 (2010).

${ }^{40}$ J. M. Falcon-Gonzalez and R. Castañeda-Priego, Phys. Rev. E 83, 041401 (2011).

${ }^{41}$ J. M. Falcon-Gonzalez and R. C. Priego, J. Chem. Phys. 133, 216101 (2010).

${ }^{42}$ H. Wennerstrom, B. Jonsson, and P. Linse, J. Chem. Phys. 76, 4665 (1982).

${ }^{43}$ M. Deserno and C. Holm, Electrostatic Effects in Soft Matter and Biophysics, NATO Science Series II: Mathematics, Physics and Chemistry Vol. 46, edited by C. Holm, P. Kekicheff, and R. Podgornik (Springer, 2001).

${ }^{44}$ S. L. Carnie and D. Y. C. Chan, Chem. Phys. Lett. 77, 437 (1981).

${ }^{45}$ R. A. Marcus, J. Chem. Phys. 23, 1057 (1955).

${ }^{46}$ F. G. Donnan, Chem. Rev. 1, 73 (1924).

${ }^{47}$ B. Zoetekouw and R. van Roij, Phys. Rev. E 73, 021403 (2006).

${ }^{48}$ R. Behera, J. Chem. Phys. 108, 3373 (1997).

${ }^{49}$ M. P. Allen and D. J. Tildesley, Computer Simulations of Liquids (Oxford University Press, Oxford, 1987).

${ }^{50}$ J. Z. Wu, D. Bratko, H. W. Blanch, and J. M. Prausnitz, J. Chem. Phys. 111, 7084 (1999).

${ }^{51}$ P. Attard, Adv. Chem. Phys. 92, 1 (1996).

${ }^{52}$ V. Lobaskin and K. Qamhieh, J. Phys. Chem. B 107, 8022 (2003).

${ }^{53}$ M. Rasa et al., J. Phys.: Condens. Matter 17, 2293 (2005).

${ }^{54}$ L. Belloni, J. Chem. Phys. 123, 204705 (2005).

${ }^{55}$ J. Dobnikar, R. Castañeda-Priego, H. von Grünberg, and E. Trizac, New J. Phys. 8, 277 (2006).

${ }^{56}$ D. Y. C. Chan, P. Linse, and S. N. Petris, Langmuir 17, 4202 (2001).

${ }^{57}$ Y. Levin and M. Fisher, Physica A 225, 164 (1996).

${ }^{58}$ J. C. Rasaiah and H. L. Friedman, J. Chem. Phys. 48, 2742 (1968).

${ }^{59}$ G. I. Guerrero-García, E. González-Tovar, and M. Chávez-Páez, Phys. Rev. E 80, 021501 (2009).

${ }^{60}$ G. I. Guerrero-García, E. González-Tovar, and M. O. de la Cruz, J. Chem. Phys. 135, 054701 (2011).

${ }^{61}$ G. I. Guerrero-García, P. González-Mozuelos, and M. O. de la Cruz, J. Chem. Phys. 135, 164705 (2011). 